\title{
Comparación de esquemas de integración 3D para elementos enriquecidos en XFEM
}

\section{Comparison of 3-D Integration Schemes for Enriched Elements in XFEM}

\author{
V.F. González-Albuixech ${ }^{1}$, E. Giner ${ }^{2}$, J.E. Tarancón ${ }^{3}$ \\ ${ }^{1}$ UC3M, Dpto. Ing. Mecánica, Universitat Politècnica de València, Valencia, España, vigonal @upv.es. \\ ${ }^{2}$ Dpto. Ing. Mecánica, Universitat Politècnica de València, Valencia, España, eginerm@mcm.upv.es. \\ ${ }^{3}$ Dpto. Ing. Mecánica, Universitat Politècnica de València UPV, Valencia, España, jetaranc@ @mcm.upv.es.
}

Recibido: may 9, 2016. Aceptado: jun16, 2016. Versión final: jun 16, 2016

\begin{abstract}
RESUMEN
El XFEM es una técnica desarrollada para la simulación numérica de problemas relacionados con la mecánica de la fractura. Este método tiene diversas ventajas, pero también aparecen ciertas cuestiones que deben ser abordadas con cuidado. La integración numérica de los elementos enriquecidos es uno de esos puntos. En este trabajo se han comparado dos técnicas posibles para realizar dicha integración, una clásica y una desarrollada especificamente para este tipo de elementos. Las diferencias en el resultado no son destacables, pero no así en la implementación. Por tanto, se recomienda el uso de la técnica clásica.
\end{abstract}

Palabras Clave: elementos enriquecidos, integración, mecánica de la fractura, XFEM.

\begin{abstract}
The XFEM is a technique developed for the numerical simulation of fracture mechanics problems. The method shows several advantages. But some questions arise that should be handled with some care. Numerical integration of the enriched elements is one of these issues. In this work we have compared two avalaible techniques for its integration. One is a classic integration scheme and another is a scheme specifically developed for this kind of elements. The differences on the results are minimal, but not in their implementation difficulty. Hence, the classical integration is recommended.
\end{abstract}

Keywords: enriched elements, fracture mechanics, integration, XFEM.

\section{INTRODUCCIÓN}

El estudio analítico del comportamiento mecánico de componentes, incluyendo el análisis del efecto de la presencia de grietas mediante el uso de la Mecánica de la Fractura Elástico Lineal (MFEL), solo puede realizarse analíticamente en casos sencillos. Los casos más complicados tienen que abordarse inevitablemente mediante técnicas numéricas que permiten obtener una solución aproximada del problema.

Una de las técnicas numéricas más consolidada y usada, tanto en ámbitos científicos como técnicos, es el método de los elementos finitos (MEF). No obstante, el MEF presenta algunos inconvenientes para la resolución de problemas donde existen singularidades, de los cuales la existencia de grietas es un caso particular. La razón es que la aproximación del MEF se basa en un desarrollo en un espacio polinómico, que puede utilizarse para estudiar, con elevada precisión, problemas cuya solución presenta un campo continuo y suave, pero presenta poca eficacia al emplearse en la descripción de comportamientos singulares.

En particular, la solución correspondiente a la existencia de la fisura presenta una discontinuidad y una región con comportamiento singular, que no se puede describir fácilmente mediante funciones polinómicas. Para conseguir una precisión adecuada en este tipo de problemas, resulta obligatorio el uso de un refinamiento local de la malla en la región donde domina la singularidad, lo que conduce a un aumento considerable de los grados de libertad (GDL). Además, la aplicación correcta del MEF a este tipo de problema, obliga a una discretización del modelo mediante una malla con topología adaptada a la geometría de la grieta, hecho que no solo añade complicaciones en el mallado inicial, sino que añade dificultades en el estudio de la propagación de la fisura, ya que se hace necesario 
realizar un remallado en cada paso de crecimiento que se adapte a la nueva configuración geométrica.

Para mejorar el comportamiento del MEF en los estudios de singularidades, especialmente en aplicaciones de la MFEL, existen varios planteamientos que permiten incluir la tendencia de los campos asintóticos en la formulación de los elementos. Entre otros se pueden nombrar [1-4]. Strang, en [5], introduce un nuevo planteamiento para este tipo de aproximaciones, que consiste en introducir términos exactos de la solución conocidos a priori en la propia formulación de la base de los elementos finitos. En el caso de la MFEL se está hablando de los términos del campo asintótico del extremo de grieta.

Belytschko y Black, en [6], introducen un método para estudiar problemas de MFEL, como la propagación de grietas. Este método, entre otras ventajas, apenas necesita realizar un remallado para el estudio del crecimiento de la grieta. La base de la técnica es el MEF, pero la aproximación es enriquecida con las mismas funciones de enriquecimiento que se usan en [7]. La topología de la malla es independiente de la grieta, pero el efecto se introduce enriqueciendo la aproximación en los elementos cercanos al frente de la grieta. Este enriquecimiento se realiza con las funciones de la solución del campo asintótico del extremo de grieta, que cumple la condición de partición de la unidad, [8-10].

Una técnica más genérica y sencilla es introducida por Moës et al. en [11]. Aquí la discontinuidad de los campos asociados a las caras de grieta se introduce usando como enriquecimiento la función de Heaviside. El enriquecimiento, tanto para el extremo de grieta como para las caras de grieta, se efectúa siguiendo unas reglas sencillas. Este método, en principio, permite obtener una mayor precisión y evita realizar mallas adaptadas a la geometría de grieta. En [12], en el estudio de grietas ramificadas, se dio a esta técnica el nombre de método de los elementos finitos extendidos o ampliados, habitualmente llamado y abreviado por XFEM, por sus siglas en inglés. Esta técnica también se ha usado en casos tridimensionales, por ejemplo en [1315] y se halla implementada en códigos comerciales como ABAQUS.

Aunque el XFEM presenta muchas ventajas, también muestra inconvenientes, como una menor velocidad de convergencia, [16-17]. Chessa atribuye este comportamiento a la falta de propiedades de la partición de la unidad en los elementos que no están totalmente enriquecidos, que se conocen como elementos de transición. Las soluciones que proponen, se basan en intentar recuperar la partición de la unidad en dichos elementos, para ello o se modifica la formulación de los elementos [18], o se introducen funciones de forma jerárquicas de orden superior [17, 19]. La zona de enriquecimiento también afecta a la velocidad de convergencia, tal como se puede observar en [19-21]. Si se usa una zona de enriquecimiento independiente de la malla, se puede conseguir mejor velocidad de convergencia. Sin embargo, el resultado es un empeoramiento en el condicionamiento de la matriz del sistema. El condicionamiento se puede mejorar eliminando problemas en la zona de transición, usando precondicionadores [20], o reduciendo el número de GDL asociados al campo asintótico [19, 21].

La integración numérica de los elementos enriquecidos es uno de los puntos en los que hay que prestar una atención especial al emplear el XFEM. La integración de dichos elementos debe ser abordada con cierto cuidado, debido a la presencia de funciones que presentan discontinuidades y singularidades. Las reglas de integración que se pueden emplear han sido consideradas en diversos trabajos [13, 20-26], que generalmente, se basan en la subdivisión del elemento en subelementos conformes con la geometría de la grieta; normalmente triángulos o cuadriláteros para dos dimensiones y en tetraedros o hexaedros para tres dimensiones, con las caras de los nuevos subelementos orientadas con la grieta.

En estos nuevos elementos se realiza una integración estándar o una integración usando una transformación de coordenadas para capturar mejor la singularidad. Hay otro tipo de técnicas, como la descrita en [27], que permiten usar una cuadratura estándar sin particionar el elemento reemplazando las funciones no integrables por polinomios equivalentes.

En este trabajo se comparan dos esquemas de integración para subelementos conformes en tres dimensiones de tipo tetraedro, de lo cual se obtiene que la integración propuesta en [25] es casi óptima, pero que resultados similares se pueden alcanzar con integraciones más sencillas de implementar que, por tanto, resultan una mejor opción.

\section{FUNDAMENTOS DEL XFEM}

El XFEM es una técnica numérica que permite el enriquecimiento local de los espacios de aproximación a partir de conceptos relacionados con la partición de la unidad. Este método es útil para resolver problemas con soluciones no suaves en pequeñas partes del dominio de cálculo, como ocurre debido a la presencia de singularidades y discontinuidades.

En comparación con el método clásico de los elementos finitos, el XFEM tiene muchas ventajas para el modelado numérico de la grieta, sobre todo si se quiere considerar su propagación. El método consiste en el enriquecimiento del modelo de EF con GDL adicionales en los nodos asociados a los elementos afectados por la grieta [13].

La discontinuidad y el comportamiento asintótico de la grieta se introducen con enriquecimientos, evitando la necesidad de modificar la malla, por lo que es posible usar solo una malla de elementos finitos para cualquier tipo de grieta y orientación. Por tanto, la misma malla es 
válida para realizar estudios de propagación. Sin embargo, la malla utilizada debe estar suficientemente refinada para la correcta descripción de la trayectoria del crecimiento de la grieta. Por tanto, es necesario un refinamiento moderado, aunque no tan fino como con el método clásico.

El XFEM utiliza enriquecimientos locales para introducir la singularidad. Así, considerando la malla de elementos finitos, los nodos cercanos al frente de grieta están enriquecidos con GDL que introducen funciones que representan el comportamiento asintótico de la grieta y la discontinuidad según la MFEL. La figura 1 representa una región de una malla formada con hexaedros lineales. Los nodos marcados con círculos están enriquecidos con GDL adicionales asociados a la discontinuidad, y los nodos marcados con cuadrados se enriquecen con GDL adicionales asociados a la singularidad.

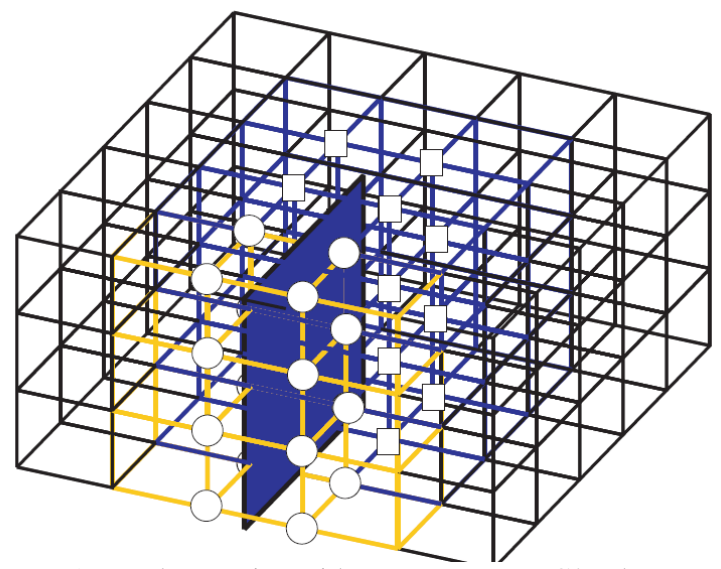

Figura 1. Nodos enriquecidos en XFEM. Círculos: nodos enriquecidos con la función de Heaviside. Elementos afectados directamente por este enriquecimiento en amarillo. Cuadrados: nodos con función de enriquecimiento de extremo de grieta. Elementos afectados por el enriquecimiento en azul. Fuente. Elaboración propia.

Los elementos que contienen al menos un nodo con enriquecimiento se conocen como elementos enriquecidos.

Los nodos con GDL asociados a la discontinuidad tienen funciones de forma que multiplican la función de Heaviside $H(\mathbf{x})$, función que cambia de signo a cada lado de la grieta. El número de nuevos GDL se corresponde con la dimensión del problema; para tres dimensiones se añaden tres nuevos GDL. Físicamente, esta función introduce la discontinuidad entre las caras de la grieta.

Los nodos que presentan el enriquecimiento correspondiente a la singularidad se enriquecen en cada dirección cartesiana mediante cuatro funciones de enriquecimiento que son capaces de reproducir los campos asociados a la singularidad del frente de grieta [15]. En tres dimensiones se enriquece, por tanto, con 12 GDL adicionales, $F_{\boldsymbol{a}}(\mathbf{x})$ :
$\left[F_{\alpha}(r, \theta), \alpha=1,4\right]=\left[\sqrt{r} \sin \frac{\theta}{2}, \sqrt{r} \cos \frac{\theta}{2}, \sqrt{r} \sin \frac{\theta}{2} \sin \theta, \sqrt{r} \cos \frac{\theta}{2} \sin \theta\right]$

donde $r, \theta$ son las coordenadas polares locales definidas en los puntos del frente de grieta.

Estas funciones reproducen el campo de desplazamientos asintótico correspondiente al comportamiento en puntos cercanos al frente de grieta según MFEL, produciendo el comportamiento singular en deformaciones y tensiones correspondientes a los puntos cercanos al frente de grieta.

Las funciones de enriquecimiento $H(\mathbf{x})$ y $F_{1}(\mathbf{x})$ son funciones discontinuas en la cara de grieta. Se elige $H(\mathbf{x})=1$ si $\mathbf{x}$ está en la parte superior de la grieta $\mathrm{y}$ $H(\mathbf{x})=-1$ en otro caso.

La discontinuidad asociada a las caras de grieta se introduce mediante la función de Heaviside en los elementos partidos por la grieta, y en el elemento enriquecido con las funciones de extremo de grieta.

La aproximación en desplazamientos utilizada es:

$\mathbf{u}_{\text {XFEM }}(\mathbf{x})=\sum_{i \in I} N_{i}(\mathbf{x}) \mathbf{u}_{i}+\sum_{i \in J} N_{i}(\mathbf{x}) H(\mathbf{x}) \mathbf{a}_{i}+\sum_{i \in K}\left\lceil N_{i}(\mathbf{x}) \sum_{\alpha=1}^{4} F_{\alpha}(\mathbf{x}) \mathbf{b}_{i \alpha}\right\rceil$

donde $I$ es el conjunto de nodos del elemento, $N_{i}(\mathrm{x})$ son las funciones de forma $\mathrm{y} \mathbf{u}_{i}$ son los GDL normales del nodo $i$ ( $\mathbf{u}_{i}$ representa el desplazamiento físico en los nodos).

Los subconjuntos $J$ y $K$ contienen los nodos enriquecidos con la función de Heaviside, $H(\mathbf{x})$, y con las funciones que reproducen el comportamiento asintótico de frente de grieta, $F_{\mathbf{a}}(\mathbf{x})$, respectivamente, además $\mathbf{a}_{i}$ y $\mathbf{b}_{i \alpha}$ son sus correspondientes GDL.

Hay que remarcar que los GDL adicionales $\mathbf{a}_{i}$ y $\mathbf{b}_{i \alpha}$ de la ecuación 2 solo se añaden en los nodos que se enriquecen. Así, si no hay enriquecimiento, la ecuación anterior se reduce a la aproximación clásica de elementos finitos, esto es:

$$
\mathbf{u}_{F E M}(\mathbf{x})=\sum_{i \in I} N_{i}(\mathbf{x}) \mathbf{u}_{i}
$$

Por tanto, el XFEM mantiene gran parte de las ventajas asociadas con el MEF.

\section{SUBDIVISIÓN E INTEGRACIÓN NUMÉRICA}

En el MEF, la integración numérica en los elementos se suele realizar mediante reglas de cuadratura de Gauss. Cuando la función a integrar es de tipo polinómico, este tipo de integración da muy buen resultado, siendo posible incluso obtener el valor exacto. Sin embargo, para el caso de funciones discontinuas o singulares pueden surgir ciertos problemas, no siendo suficiente la estrategia de usar cuadraturas de mayor orden. Una 
buena opción para estos casos es la realización de subdivisiones donde se mantiene un bajo orden de cuadratura [28].

En el estudio de comportamiento de grietas mediante el MEF, la malla se adapta a la geometría de la grieta, pudiéndose aplicar sin problemas los esquemas de integración basados en el método de Gauss-Legendre. Si el problema es abordado usando el XFEM, la malla no se adapta a la geometría de la grieta y es necesario integrar funciones no continuas o singulares. Esta integración numérica ha sido objeto de distintos trabajos [13, 20-26].

Los elementos de la aproximación XFEM que involucran solo funciones de forma estándar de elementos finitos, es decir, aquellos elementos en que no se realiza enriquecimiento, requieren únicamente una regla de integración normal, de tipo Gauss-Legendre, como se muestra en [29-30].

En los elementos intersectados por la grieta $\mathrm{y}$ enriquecidos con la función que representa la discontinuidad es necesario unir adecuadamente las contribuciones de ambos lados de la grieta y el uso de una regla de integración estándar de Gauss-Legendre es incorrecto, ya que la malla y la grieta están orientados arbitrariamente entre sí y además la función a integrar presenta discontinuidad.

Es necesario modificar la forma en que se realiza la cuadratura en el elemento, con tal de que la forma débil esté bien representada, para lo que se integra el dominio elemental mediante la construcción de subdominios.

Moës, en [13], define los subdominios del elemento como la suma de subpolígonos cuyos contornos se alinean con la geometría de la grieta, que se convierte en frontera en la subdivisión junto con el contorno del elemento. Estos subpolígonos son necesarios únicamente para la integración numérica y no conllevan grados de libertad adicionales.

$\mathrm{Si}$ al integrar la función de enriquecimiento $H(\mathbf{x})$, el resultado es indistinguible de una función constante pueden aparecer singularidades en el sistema de ecuaciones. Para evitar este fenómeno, Daux et al, en [31], no enriquecen los nodos en los que el soporte está casi por completo a un solo lado de la grieta.

La elección de estos subpolígonos se realiza desde un punto de vista de simplicidad en su construcción. En los elementos bidimensionales se suele usar una subdivisión en triángulos, de manera que la grieta coincida con alguno de sus lados. En los elementos tridimensionales, como es nuestro caso de estudio, se subdividen en tetraedros.

El algoritmo que se utiliza para la subdivisión a partir de las regiones obtenidas en el corte del elemento por la grieta es introducido en [32], este produce triángulos - 2-D - o tetraedros - 3-D - de forma que la superficie de grieta se corresponde con un lado de los subdominios y el frente de grieta coincide con alguna de las aristas o contiene un vértice. Un ejemplo del resultado de dicho algoritmo para una malla bidimensional de triángulos se puede observar en la figura 2 .

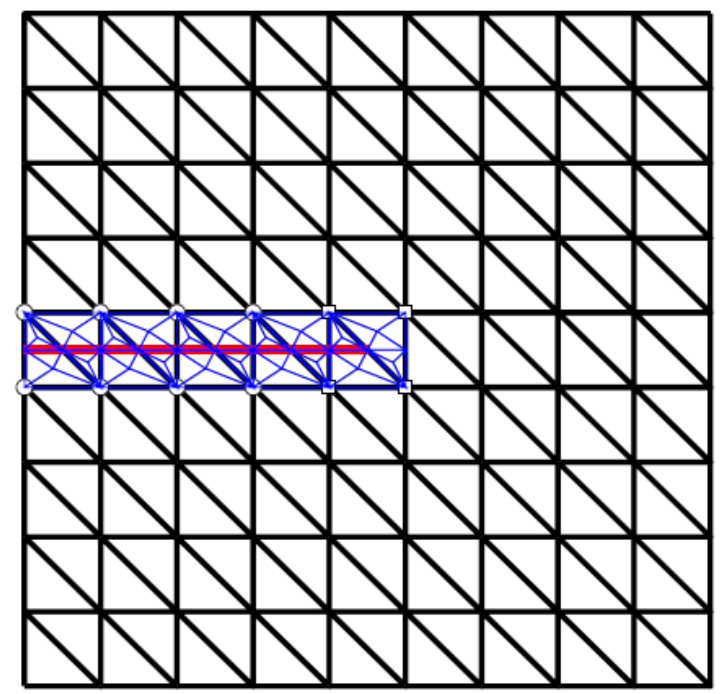

Figura 2. Ejemplo de subdivisión de elementos. Fuente. Elaboración propia.

Los elementos que contienen el frente de grieta, además de introducir la discontinuidad, poseen términos que se corresponden con funciones singulares. El uso de cuadraturas Gauss-Legendre normales para su integración produce resultados no óptimos.

Es decir, es necesario usar un elevado orden de cuadratura o añadir subdivisiones para aumentar el número de puntos de integración. Sin embargo, estas estrategias no son las más adecuadas. Laborde, en [21], propone usar un esquema basado en una transformación de coordenadas de los puntos de cuadratura, de manera que queden concentrados alrededor de la singularidad siguiendo una distribución polar centrada en el extremo de grieta, procedimiento conocido por el nombre de integración «casi-polar», que es fácil de implementar y da buenos resultados como puede observarse en [19, 22]. Pero no existen apenas estudios para tres dimensiones. 


\subsection{Integración «casi-polar» 3D de Park}

Existe una implementación de la técnica de integración «casi-polar» en tres dimensiones [25]. Aquí se parte de las reglas de integración sobre un prisma. El prisma se transforma en un prisma de referencia de donde se construye el tetraedro de integración. El esquema de transformación se presenta en la figura 3.6. La transformación no trivial, $T q$, del prisma de referencia en el tetraedro se encuentra en la ecuación 3.

$$
\begin{gathered}
\bar{r}=\bar{\rho} \cos ^{2} \bar{\theta} \\
\bar{s}=\bar{\rho} \operatorname{sen}^{2} \bar{\theta} \\
\bar{t}=\overline{t^{\prime}}
\end{gathered}
$$

Un ejemplo de la distribución de los puntos de cuadratura de dicho método se presenta en las figuras 3 y 4. Hay que destacar que este tipo de integración es relativamente complicado y que incluso en el artículo que lo presentan hay ocasiones en que la regla no funciona, al aparecer puntos de integración en el exterior del subelemento.

\subsection{Integración basada en el método clásico de Stroud}

Una opción sencilla para la integración es usar un alto orden de integración en tetraedros, a partir de un hexaedro colapsado adecuadamente, como se muestra en la figura 5. Estas reglas de integración son clásicas y fáciles de encontrar en la bibliografía, por ejemplo en [33] y posteriormente en [34]. Para aprovechar el método de colapso de Stroud en un tetraedro seguimos la siguiente regla: la arista donde se colapsa el hexaedro se hace coincidir con la arista que comparte el tetraedro con el frente de grieta, y el nodo donde se colapsan más nodos del hexaedro se hace coincidir con uno de los vértices que coinciden con el frente de la grieta.

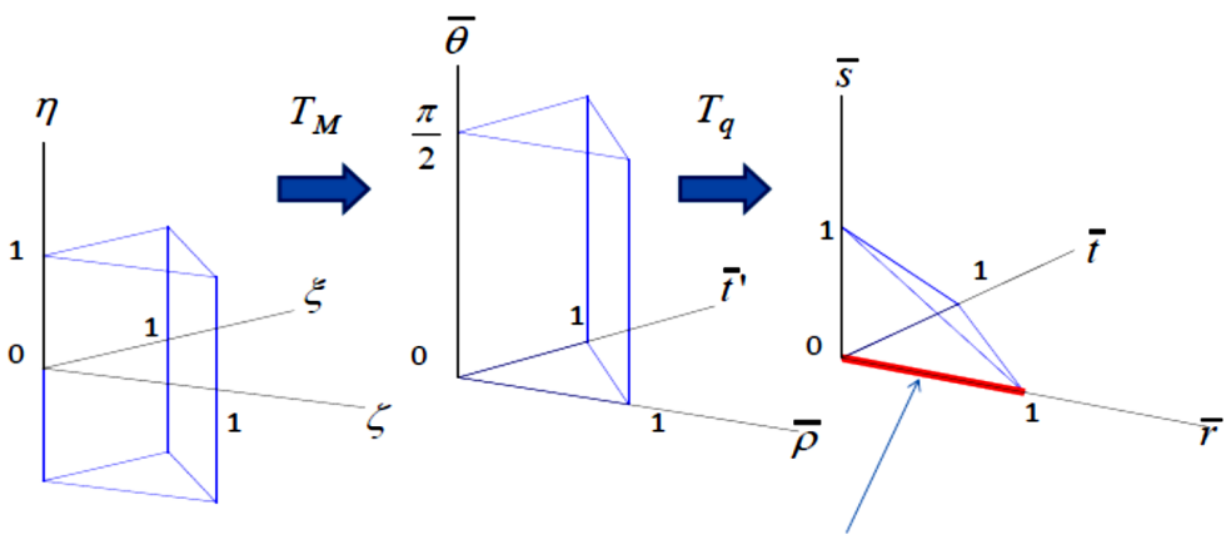

Frente de grieta

Figura 3. Transformación de dominios para la integración «casi-polar» de Park. Fuente. Elaboración propia

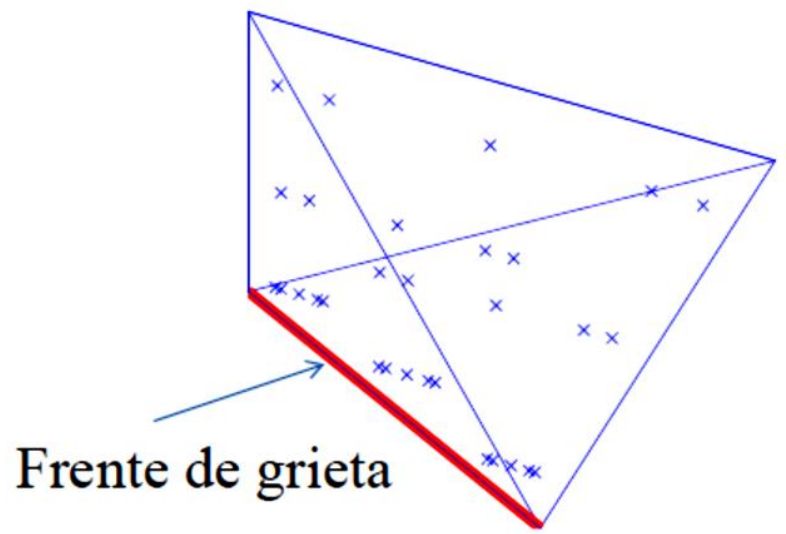

Figura 4. Ejemplo de distribución de los puntos de cuadraturas «casi-polar» de Park. Fuente. Elaboración propia. 


\section{Colapso de los nodos del hexaedro}

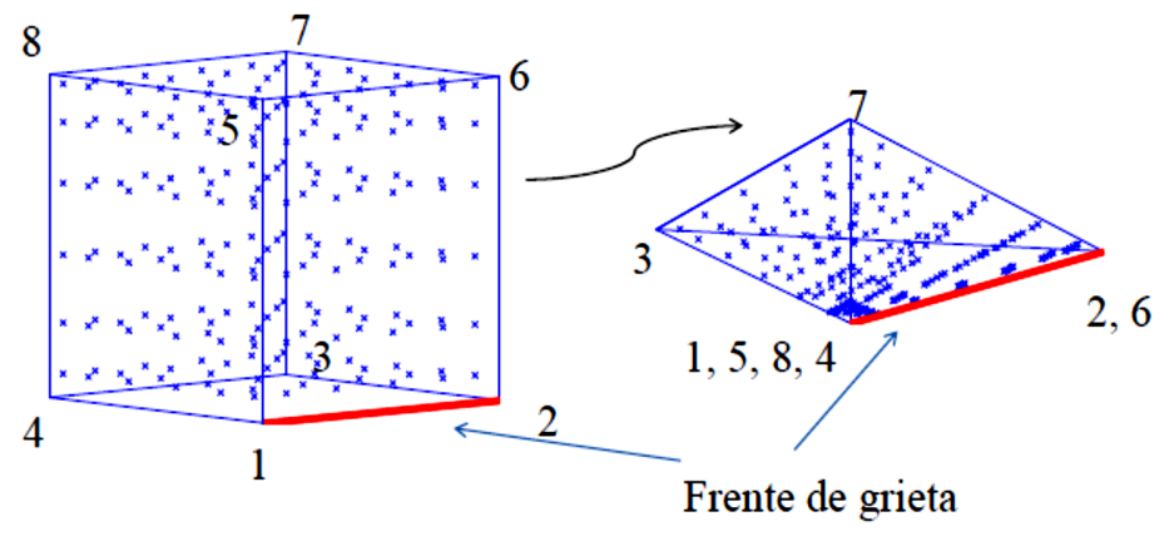

Figura 5. Distribución de los puntos de cuadraturas de Stroud. Fuente. Elaboración propia.

Además existen otros métodos, como los introducidos en [20, 35]. Estos métodos no son considerados en este estudio, ya que se alejan de las reglas clásicas de integración.

\section{VERIFICACIÓN NUMÉRICA}

Uno de los mayores problemas para realizar verificaciones numéricas en estudios tridimensionales es la falta de soluciones analíticas para casos finitos. Existen soluciones correspondientes a situaciones infinitas, pero que imponen limitaciones en el posible modelado numérico. Por ejemplo, si no se pueden imponer las condiciones de contorno adecuadas, se debe utilizar una aproximación a dominio infinito introducido por un dominio muy grande en comparación a las dimensiones de la grieta.

Otra opción es imponer las tracciones exactas o desplazamientos exactos en la posición de un contorno. Los ejemplos que se suelen usar para verificar los métodos en casos tridimensionales suelen corresponderse con casos bidimensionales extruidos, que - exceptuando en algunos casos patológicos- son lo bastante genéricos para permitir la realización de un análisis tridimensional. Un ejemplo de este tipo se utiliza para comparar las reglas de integración.

El problema considerado tiene una solución con parte suave y singular. Se le conoce como problema de Westergaard. En el problema se considera una grieta de longitud $2 a$ en una placa infinita sometida a tracción biaxial uniforme combinada con carga tangencial en el infinito, como se muestra en la figura 6 . Este problema tiene la siguiente expresión a primer orden en los campos de desplazamiento:

$$
\left\{\begin{array}{l}
u_{1} \\
u_{2}
\end{array}\right\}=\frac{K_{1}}{2 \mu} \sqrt{\frac{r}{2 \pi}}\left\{\begin{array}{c}
\cos \frac{\theta}{2}\left(\kappa-1+2 \operatorname{sen}^{2} \frac{\theta}{2}\right) \\
\operatorname{sen} \frac{\theta}{2}\left(\kappa+1-2 \cos ^{2} \frac{\theta}{2}\right)
\end{array}\right\}+\frac{K_{\text {II }}}{2 \mu} \sqrt{\frac{r}{2 \pi}}\left\{\begin{array}{c}
\operatorname{sen} \frac{\theta}{2}\left(\kappa+1+2 \cos ^{2} \frac{\theta}{2}\right) \\
-\cos \frac{\theta}{2}\left(\kappa-1-2 \operatorname{sen}^{2} \frac{\theta}{2}\right)
\end{array}\right\}
$$

donde $u_{i}$ es el desplazamiento en la dirección $x_{i}, K_{\mathrm{I}}$ y $K_{\mathrm{II}}$ son los factores de intensidad de tensiones en modo I y II respectivamente, $r$ y $\theta$ las coordenadas polares relativas al extremo de grieta, $\mu$ es el módulo de rigidez a cizalladura y $\kappa$ la constante de Kolosov, definidas en función de los parámetros del material como:

$$
\mu=\frac{E}{2(1+v)}, \quad \kappa=3-4 v
$$

A partir de esta definición se pueden imponer las condiciones de contorno exactas en un modelo finito extruido - similar a la presentada en la figura 1-. El modelo de elementos finitos usado se corresponde con un cubo finito de longitudes totales de lado en los ejes $X$ y $Y$ de $2 a$ y en el eje $Z$ (en la dirección de la grieta) $a$. La secuencia de mallas regulares, siguiendo la construcción empleada en la fig. 1 y usada en este análisis, se corresponde con los siguientes tamaños de elemento $h=a(1 / 8,1 / 16,1 / 20,1 / 32,1 / 40)$.

Los elementos finitos, y los tetraedros resultados de la subdivisión que no necesitan una integración especial, se realizan con una integración con regla de Gauss normal de 64 puntos. La integración de los tetraedros que contienen singularidad se realiza mediante un hexaedro colapsado de 64 puntos, siguiendo las reglas clásicas preentadas por [33-34] o mediante una regla de 42 puntos según el esquema de [25]. 


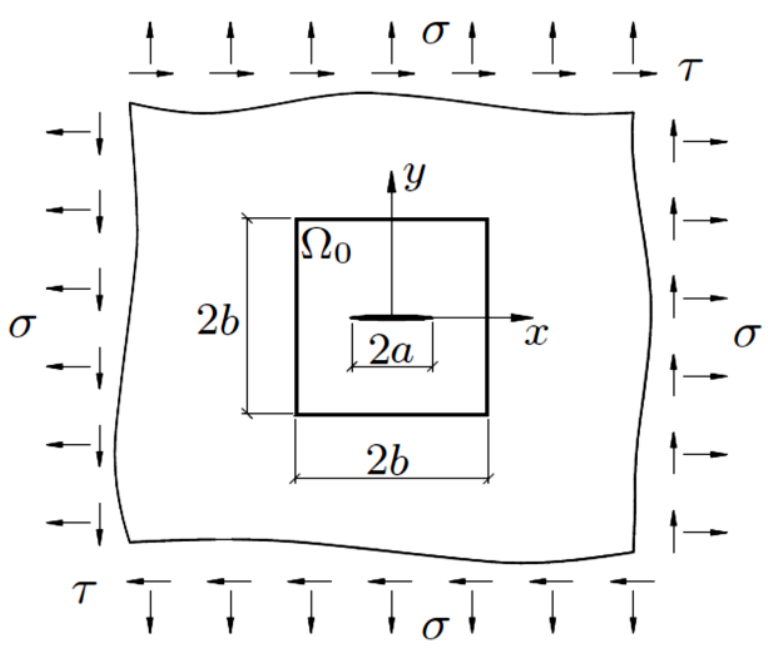

Figura 6. Definición geométrica del problema de Westergaard en dos dimensiones. Fuente. Elaboración propia.

Solo se analizan los casos en que la solución presenta $K_{\mathrm{I}}$ o $K_{\mathrm{II}}$, lo que permite estudiar el comportamiento de las reglas de integración adaptadas al extremo de grieta. Para ello, se consideran varias mallas con hexaedros regulares y distinto tamaño de se presenta en la figura 7 para $K_{\mathrm{I}}$ y en la figura 8 para el $K_{\text {II. }}$.

La velocidad de convergencia óptima en norma enérgetica en el estudio propuesto es 0,5 , siendo los resultados muy similares a ese valor. Por tanto, el comportamiento obtenido con ambas rutinas de integración es correcto. Sin embargo, el esquema clásico consigue mayor precisión y es mucho más fácil de implementar. Además, al disponer de varias decadas de uso, se puede aceptar como sobradamente comprobado, por tanto, se puede considerar como la mejor opción.

\section{CONCLUSIONES}

El XFEM es una técnica desarrollada para la simulación numérica de problemas relacionados con la mecánica de la fractura. El método se basa en la introducción de los comportamientos correspondientes a las singularidades y discontinuidades asociadas a la presencia de la grieta mediante un enriquecimiento de los GDL de una malla de elementos finitos.

Sin embargo, la presencia de dicho enriquecimiento hace que la integración de los elementos enriquecidos requiera un tratamiento especial, sobre todo en los elementos donde se considera el comportamiento correspondiente a la singularidad.

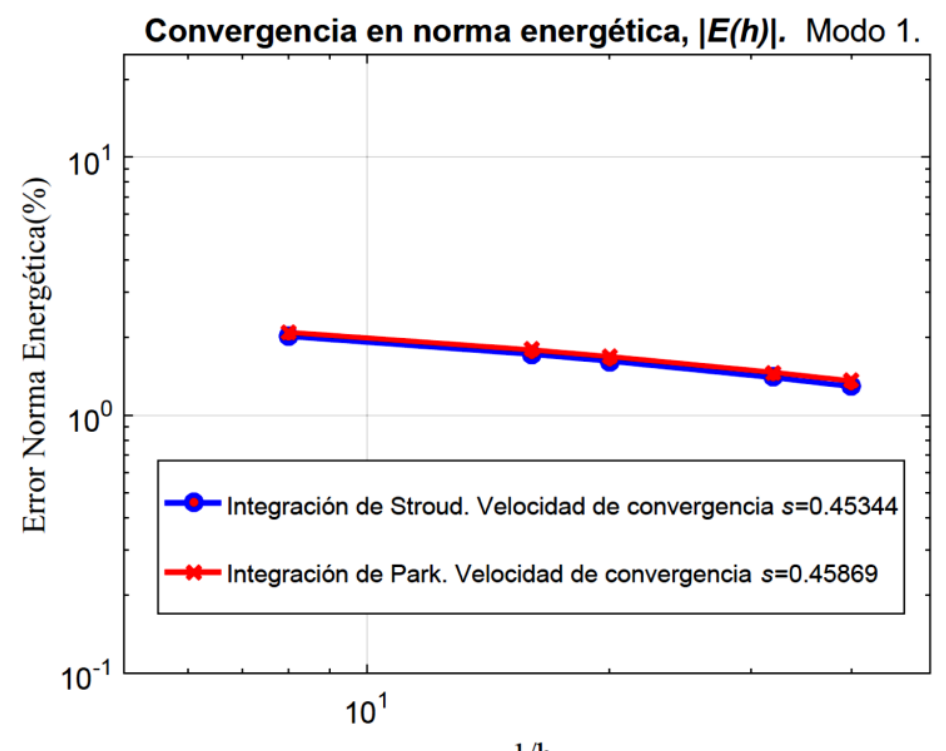

$1 / \mathrm{h}$

Figura 7. Convergencia en energía para el problema Westergaard con solo $K_{\mathrm{I}}$. Fuente. Elaboración propia.

elemento, que permiten estudiar la evolución del error en la energía con la inversa del tamaño de elemento y se hace una comparación de la convergencia en norma energética, calculada mediante la adición en todo el modelo de los resultados de la integración de la energía para cada elemento, para los dos esquemas de integración de la singularidad considerados. El resultado
En este trabajo se ha comparado el comportamiento de dos esquemas de integración, usando un ejemplo sencillo con solución análitica. Uno de dichos esquemas está basado en métodos clásicos $[33,34]$ y es usado en la integración de tetraedros y el otro fue desarrollado por [25], para la integración de este tipo de problemas. 
Convergencia en norma energética,|E(h)|. Modo 2.

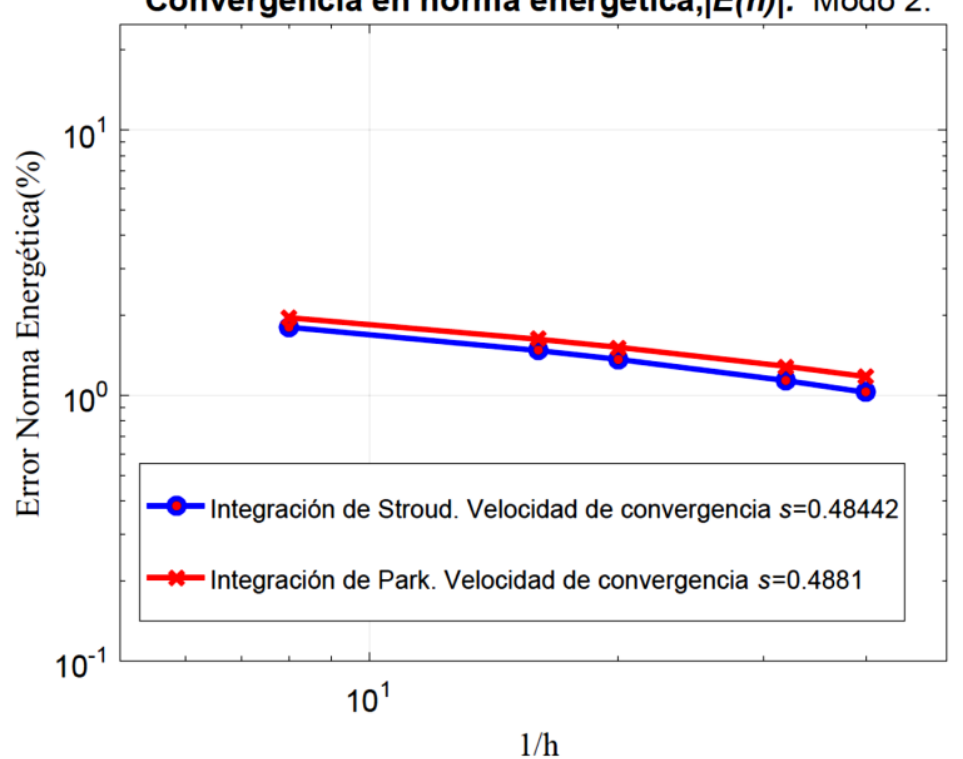

Figura 8. Convergencia en energía para el problema Westergaard con solo $K_{\text {II }}$.

Las diferencias en el resultado son escasas y ambas técnicas presentan casi la misma velocidad de convergencia, siendo en los dos casos prácticamente la óptima. Tampoco se observan diferencias importantes en su nivel de error, aunque quizás la técnica clásica es ligeramente más precisa, aunque esto puede ser debido al mayor orden de integración empleado. Sin embargo, la implementación de ambas técnicas difiere mucho en su dificultad y, además, la técnica clásica dispone de la ventaja de la robustez al haber sido verificada durante décadas. Más aún, si se tiene en cuenta que los mismos autores de la técnica desarrollada especialmente para XFEM reconocen que alguno de sus esquemas da problemas.

Por tanto, la técnica basada en la integración clásica de Stroud constituye una mejor opción para la implementación de XFEM en un uso ingenieril.

\section{RECOMENDACIONES}

Un posible punto a tener en cuenta es el estudio y comparación de distitnos ordenes de integración, así como la extensión a elementos de orden cuadrático, o mayor. Asimismo, sería interesante considerar la verificación en casos con grietas curvas y no planas, y estudiar el comportamiento en elementos con tamaño no regular.

Todas estas situaciones pueden suponer limitaciones tanto en la técnica de integración usada en los elementos enriquecidos, como en el mismo XFEM. Por tanto, los resultados obtenidos al estudiar este tipo de casos deben ser considerados con cuidado.

\section{REFERENCIAS}

[1] R. Barsoum, "Triangular quarter-point elements as elastic and perfectly plastic crack tip elements," International Journal of Numerical Methods in Engineering, vol. 11, núm. 1, pp. 85-98, 1977. DOI: $10.1002 / \mathrm{nme} .1620110109$

[2] R. Gallagher, "A review of finite element techniques in fracture mechanics," lst Conference on Numerical Methods in Fracture Mechanics, (Swansea, R.U.), 1978, pp. 1-25.

[3] A.J. Fawkes, D.R.J. Owen y A.R. Luxmoore. "An assessment of crack tip singularity models for use with isoparametric elements," Engineering Fracture Mechanics, vol. 11, núm. 1, pp. 143-159, 1979. DOI:10.1016/0013-7944(79)90035-3

[4] Y.W. Kwon y J.E. Akin, "Development of a derivative singular element for application to crack propagation problems," Computers \& Structures, vol. 31, núm. 3, pp. 467-471, 1989.

[5] G. Strang, G.J. Fix, An Analysis of the Finite Element Method, Upper Saddle River, EEUU: Prentice-Hall, 1973.

[6] T. Belytschko y T. Black, "Elastic crack growth in finite elements with minimal remeshing," International Journal of Numerical Methods in Engineering, vol. 45, núm. 5, pp. 601-620, 1999. DOI: $\quad$ 10.1002/(SICI)1097-0207(19990620)45:5 $<601::$ AID-NME598>3.0.CO;2-S

[7] M. Fleming, et al. "Enriched element-free galerkin methods for crack-tip field." International Journal of Numerical Methods in engineering, vol. 40, núm. 8, pp. 1483-1504, abr. 1997. 
[8] I. Babuška, G. Caloz, J. Osborn, "Special finite element methods for a class of second order elliptic problems with rough coefficients," SIAM Journal on Numerical Analysis, vol. 31, núm. 4, pp. 945-981, ago. 1994. http://www.jstor.org /stable/2158110

[9] J.M. Melenk e I. Babuška, "The partition of unity finite element method: Basic theory and applications," Computer Methods in Applied Mechanics and Engineering, vol. 39, núm. 1-4, pp. 289-314, dic. 1996. DOI: 10.1016/S00457825(96)01087-0.

[10] I. Babuška y J. M. Melenk, "The partition of unity method," International Journal of Numerical methods in engineering, vol. 40, núm. 4 pp. 727758, 1997. DOI: 10.1002/(SICI)1097-0207 (19970228)40:4<727::AID-NME86>3.0.CO;2-N.

[11] N. Moës, J. Dolbow y T. Belytschko, "A finite element method for crack growth without remeshing," International Journal for Numerical Methods in Engineering, vol. 46, núm. 1, pp. 131150, 1999. DOI: 10.1002/(SICI)1097-0207 (19990910)46:1<131::AID-NME726>3.0.CO;2-J.

[12] C. Daux et al. "Arbitrary branched and intersecting cracks with the extended finite element method," International Journal of Numerical Methods in Engineering, vol. 48, núm. 12, pp. 1741-1760, ago. 2000. DOI: 10.1002/1097-0207(20000830) 48:12<1741::AID-NME956>3.0.CO;2-L.

[13] N. Moës, A. Gravouil y T. Belytschko, "Nonplanar 3d crack growth by the extended finite element and level sets-Part I: Mechanical model," International Journal for Numerical Methods in Engineering, vol. 53, núm. 11, pp. 2549-2568, abr. 2002. DOI: $10.1002 / \mathrm{nme} .429$.

[14] A. Gravouil, N. Moës y T. Belytschko, "Nonplanar 3D crack growth by the extended finite element and level sets-Part II: Level set update," International Journal for Numerical Methods in Engineering, vol. 53, núm. 11, pp. 2569-2586, abr. 2002. DOI: $10.1002 / \mathrm{nme} .430$.

[15] N. Sukumar et al., "Extended finite element method for three-dimensional crack modelling," International Journal of Numerical Methods in Engineering, vol. 48, núm. 11, pp. 1549-1570, 2000. DOI: $10.1002 / 1097-0207(20000820) 48: 11$ $<1549::$ AID-NME955>3.0.CO;2-A.

[16] F. L.Stazi, E. Budyn, J. Chessa et al. "An extended finite element method with higher-order elements for curved cracks," Computational Mechanics, vol. 31, núm. 1 , pp. 38-48, may. 2003. DOI: 10.1007 /s00466-002-0391-2.
[17] J. Chessa, H. Wang y T. Belytschko, "On the construction of blending elements for local partition of unity enriched finite elements," International Journal for Numerical Methods in Engineering, vol. 57, núm. 7, pp. 1015-1038, jun. 2003. DOI: $10.1002 / \mathrm{nme} .777$.

[18] T.P. Fries, "A corrected XFEM approximation without problems in blending elements," International Journal for Numerical Methods in Engineering, vol. 75, núm. 5, pp. 503-532, jul. 2008. DOI: $10.1002 / \mathrm{nme} .2259$.

[19] J.E. Tarancón et al., "Enhanced blending elements for XFEM applied to linear elastic fracture mechanics," International Journal for Numerical Methods in Engineering, vol. 77, núm. 1, pp. 126148, ene. 2009. DOI: $10.1002 / n m e .2402$.

[20] E. Béchet et al., "Improved implementation and robustness study of the X-FEM for stress analysis around cracks," International Journal of Numerical Methods in Engineering, vol. 64, núm. 8, pp. 1033-1056, oct. 2005. DOI: 10.1002 /nme.1386.

[21] P. Laborde et al., "High-order extended finite element method for cracked domains," International Journal of Numerical Methods in Engineering, vol. 64, núm. 3, pp. 354-381, sep. 2005. DOI: $10.1002 / \mathrm{nme} .1370$.

[22] N. Sukumar y J. H. Prevost, "Modeling quasistatic crack growth with the extended finite element method part I: Computer implementation," International Journal of Solids and Structures, vol. 40, núm. 26, pp. 7513-7537, dic. 2003. DOI: 10.1016/j.ijsolstr.2003.08.002

[23] B.L. Karihaloo y Q.Z. Xiao, "Modelling of stationary and growing cracks in FE framework without remeshing: a state-of-the-art review," Computers \& Structures, vol. 81, núm. 3, pp. 119129, feb. 2003. DOI: $10.1016 / S 0045-7949(02)$ 00431-5.

[24] Q. Xiao y B. Karihaloo, "Improving the accuracy of XFEM crack tip fields using higher order quadrature and statically admissible stress recovery," International Journal of Numerical Methods in Engineering, vol. 66, núm. 9, pp. 1378-1410, may. 2006. DOI: 10.1002/nme.1601.

[25] K. Park et al., "Integration of singular enrichment functions in the generalized/extended finite element method for three-dimensional problems," International Journal for Numerical Methods in Engineering, vol. 78, núm.10, pp. 1220-1257, jun. 2009. DOI: $10.1002 / \mathrm{nme} .2530$. 
[26] J. Dolbow, N. Moës y T. Belytschko, “An extended finite element method for modelling crack growth with frictional contact," Computer Methods in Applied Mechanics and Engineering, vol. 190, núm. 51-52, pp. 6825-6846, oct. 2001. DOI: $10.1016 / \mathrm{S} 0045-7825(01) 00260-2$.

[27] J. Chessa, H. Wang y T. Belytschko, "On the construction of blending elements for local partition of unity enriched finite elements," International Journal for Numerical Methods in Engineering, vol. 57, núm. 7, pp. 1015-1038, jun. 2003. DOI: 10.1002/nme.777.

[28] A. Quarteroni, R. Sacco y F. Saleri, Numerical mathematics, Berlin, Alemanía: Springer, 2007.

[29] T. Strouboulis, I Babuška y K. Copps, "The design and analysis of the generalized finite element method," Computer Methods in Applied Mechanics and Engineering, vol. 181, núm. 1-3, pp. 43-69, ene. 2000. DOI: $10.1016 /$ S0045-7825 (99)00072-9.

[30] T. Strouboulis, K. Copps y I. Babuška, "The generalized finite element method: an example of its implementation and illustration of its performance," International Journal of Numerical Methods in Engineering, vol. 47, núm. 8, pp. 1401-1417, mar. 2000. DOI: 10.1002/(SICI)10970207(20000320)47:8<1401::AID-NME835>3.0 .CO;2-8.

[31] C. Daux et al., "Arbitrary branched and intersecting cracks with the extended finite element method," International Journal of Numerical Methods in Engineering, vol. 48, núm.12, pp. 1741-1760, ago. 2000. DOI: 10.1002/1097-0207(20000830)48:12<1741::AIDNME956>3.0.CO;2-L.

[32] Element partitioning code in 2-D and 3-D for the extended finite element method. 2016. Disponible en: http://dilbert.engr.ucdavis.edu/ suku/xfem

[33] A.H. Stroud, Approximate Calculation of Multiple Integrals, Upper Saddle River, EEUU: PrenticeHall, 1971.

[34] P. Keast, "Moderate-degree tetrahedral quadrature formulas," Computer Methods in Applied Mechanics and Engineering, vol. 55, núm. 3, pp. 339-348, may. 1986. DOI:10.1016/0045-7825(86) 90059-9.

[35] G. Ventura, "On the elimination of quadrature subcells for discontinuous functions in the extended finite-element method," International Journal of Numerical Methods in Engineering, vol. 66, núm. 5, pp. 761-795, abr. 2006. DOI: 10.1002/nme. 1570 